Z Herz- Thorax- Gefäßchir 2022 $\cdot 36: 32$ https://doi.org/10.1007/s00398-021-00480-9 Angenommen: 6. Dezember 2021

(C) The Author(s), under exclusive licence to Springer Medizin Verlag GmbH, ein Teil von Springer Nature 2021

\section{Vor 50 Jahren im Brennpunkt}

\author{
Markus K. Heinemann
}

Klinik und Poliklinik für Herz- und Gefäßchirurgie, Universitätsmedizin Mainz, Mainz, Deutschland
In diesem Jubiläumsheft zum 50-jährigen Bestehen der Deutschen Gesellschaft für Thorax-, Herz- und Gefäßchirurgie (DGTHG) lag es nahe, die Im-BrennpunktBeiträge auf Besprechungen von Artikeln zu beziehen, die im Gründungsjahr publiziert worden sind. Zum Glück konnten Autoren gewonnen werden, die die Zeit seitdem als aktive Herzchirurgen miterlebt und mitgestaltet haben.

Der erste Beitrag schlägt eine Brücke von der Grundlagenforschung zur Klinik. Aus Köln wurden „Klinische Erfahrungen mit der Kardioplegie nach Bretschneider" berichtet. Claus Preuße hat maßgeblich zur Entwicklung dieser Protektionslösung beigetragen, sodass es nahelag, ihn um eine kritische Würdigung zu bitten. Er hat den Artikel sogar noch um ein von Bretschneider handgeschriebenes „Kochrezept“ ergänzt.

\section{\) Aktuelles von vor 50 Jahren}

In der zweiten Publikation wird über "Spätergebnisse nach Aortenklappenersatz" aus Göttingen berichtet, genauer über „150 Klappenersatzoperationen mit der StarrEdwards-Prothese". Armin Welz hat für uns die Entwicklung dieser damals bahnbrechenden Technik, und was inzwischen aus ihr geworden ist, in den nötigen historischen Rahmen gestellt.

Beide Besprechungen lassen den Enthusiasmus der Autoren spüren, die Entwicklung der Herzchirurgie in Deutschland noch einmal exemplarisch erzählen zu dürfen. In diesem Sinne wünsche ich viel Freude beim Lesen gelebter Geschichte.

\section{Ihr}

Markus Heinemann

\section{Korrespondenzadresse}

Prof. Dr. Markus K. Heinemann

Klinik und Poliklinik für Herz- und

Gefäßchirurgie, Universitätsmedizin Mainz Langenbeckstr. 1, 55131 Mainz, Deutschland mheinema@uni-mainz.de

Interessenkonflikt. M. K. Heinemann gibt an, dass kein Interessenkonflikt besteht. 